\title{
Drug Repurposing in Antiviral Research: A Current Scenario
}

\author{
Divyabharathi Mani ${ }^{1}$, Ashish Wadhwani ${ }^{1, *}$, Praveen Thaggikuppe Krishnamurthy ${ }^{2}$ \\ 'Department of Pharmaceutical Biotechnology, JSS Academy of Higher Education and Research, College of Pharmacy, Ooty, Tamil Nadu, INDIA. \\ ${ }^{2}$ Department of Pharmacology, JSS Academy of Higher Education and Research, College of Pharmacy, Ooty, Tamil Nadu, INDIA.
}

\begin{abstract}
Better understand the unexplored parts of the drugs you know than the drugs unidentified. Drug repurposing is a promising, fast and cost-effective method that can overcome traditional de novo drug discovery and development challenges of targeting various diseases and disorders. Drug repurposing, the process of identifying new uses for the existing or candidate drugs is an effective strategy for drug discovery in various diseases. Identifying new drugs and new target is significant in today's world with a new disease emerging every day. With increase in number of the emerging viral infections day by day, the targeted therapies for those are not discovered in parallel. The drug repurposing approach has given many promising drug candidates for various viral infectious diseases like Ebola, ZIKA, dengue, influenza, HIV, HSV, CMV infections and various other infectious diseases. The emergence of resistance to existing antiviral drugs and re-emerging viral infections are the biggest challenges in the antiviral drug discovery. The drug repurposing approach is an assuring strategy in finding new
\end{abstract}

potential antiviral agents within a short span of time to overcome the challenges in antiviral therapy. In this review, we describe the most promising results of the drug repurposing approach in the treatment of various infectious diseases.

Key words: Antiviral, Drug resistance, Re-emerging viral infections, Repurposing

\section{Correspondence}

Dr. Ashish Wadhwani, Department of Pharmaceutical Biotechnology, JSS Academy of Higher Education and Research, College of Pharmacy, Ooty - 643001, Tamil Nadu, INDIA.

Phone: +91-8903638815

Email: adwadhwaniad@gmail.com

DOI: 10.5530/jyp.2019.11.26

\section{INTRODUCTION}

Viruses are a family of large group of pathogens which causes severe infectious diseases. In past 30 years, many antiviral agents which targets viral proteins or host factors have been developed successfully. The chronic viral infectious diseases, such as human immunodeficiency virus (HIV), influenza virus and hepatitis $\mathrm{C}$ virus (HCV); the re-emergence of many new infections, like picorna viruses and coronaviruses; and, also resistance developed to the available antiviral drugs are the main reasons for increased demand for new antiviral agents. The growing knowledge in understanding the molecular mechanisms of the infection has provided great insight for the discovery of new antiviral agents which will target different viral proteins or host factors. The need for new antiviral drugs in treatment of chronic infectious diseases and the emerging more efficient new viruses acts as motivation for research to find more new targets and mechanisms for the development of new antivirals. ${ }^{1}$

From 2012 to 2017, only 12 new antiviral drugs have been approved by the Food and Drug Administration (FDA) in the USA, of which 8 are for the treatment of hepatitis $\mathrm{C}$ virus (HCV)-related pathologies and 2 are combinations of anti-human immunodeficiency virus (HIV) drugs (www.fda.gov). At the same time, the governments and the World Health Organization (WHO) faces the global threat of many emerging and re-emerging viruses, which are responsible for alarming out breaks in recent years. These infectious diseases still lack specific treatment, such as Zika virus (ZIKV), Ebola virus (EBOV) and Middle East respiratory syndrome corona virus (MERS-CoV) and many other emerging viruses. ${ }^{2}$

\section{DRUG RESISTANCE}

Drug resistance is defined as a reduced susceptibility to a drug in a laboratory culture system and is expressed as an altered $\mathrm{IC}_{50}$ or $\mathrm{IC}_{90}$ (drug concentration required to inhibit viral growth by $50 \%$ or $90 \%$ respectively).
The Human Immunodeficiency Virus (HIV) has developed resistance to nearly all the classes of antiretroviral drugs which includes the nonnucleoside reverse transcriptase inhibitors, the protease inhibitors and the nucleoside analogue reverse transcriptase inhibitors. ${ }^{3}$ The Herpes simplex virus infections has also been reported to have developed resistance against nucleoside analogue like acyclovir primarily among immune compromised patients and particularly allogeneic bone marrow transplant patients. ${ }^{4}$ The CMV resistant to many of the antiviral agents is a well-documented complication in case of long-term therapy with those Antiviral agents. This problem has been observed mostly in immune compromised patients with AIDS and CMV retinitis, in whom drugresistant $\mathrm{CMV}$ infections have been associated with clinical progression and therapeutic failure. ${ }^{5}$

\section{DRUG REPURPOSING}

The process of finding new uses outside the scope of the original medical indication for existing drugs is also known as redirecting, repurposing, repositioning and re-profiling. The problem in productivity and worldwide pressure on increasing prices and the growing number of regulatory hurdles one has to pass through has compelled many drug developers to find new uses and new targets as more improvised version of the already existing drugs. ${ }^{6}$ The Traditional drug discovery pathway is complex, expensive and time consuming processes. The drug repurposing approach significantly reduces the time and cost for the drug discovery for infectious diseases. The emergence of resistant viruses and the untoward side effects limits the efficacy of the developed drugs targeting the viral proteins and host factors. ${ }^{7}$ The drug repurposing approach which is the process of finding new indications for existing FDA-approved drugs, is a promising alternative to accelerate the process of drug development for infectious 


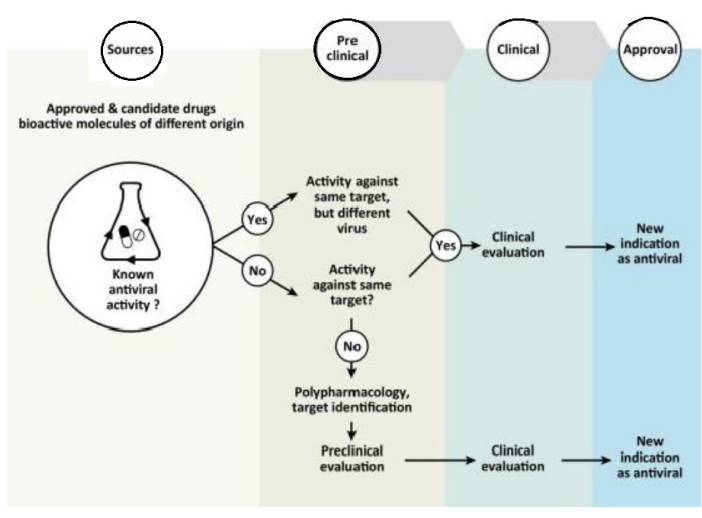

Figure 1: Repurposing of existing drugs can be pursued by these strategies. If the molecule has a previous known antiviral activity against other viruses can be hypothesized and tested based on similarity of the target or dependence on common pathways. In the absence of previously identified antiviral activity, the repurposed molecule can either act against the same target or show poly-pharmacology or interfere with a different function (cellular or viral). ${ }^{2}$

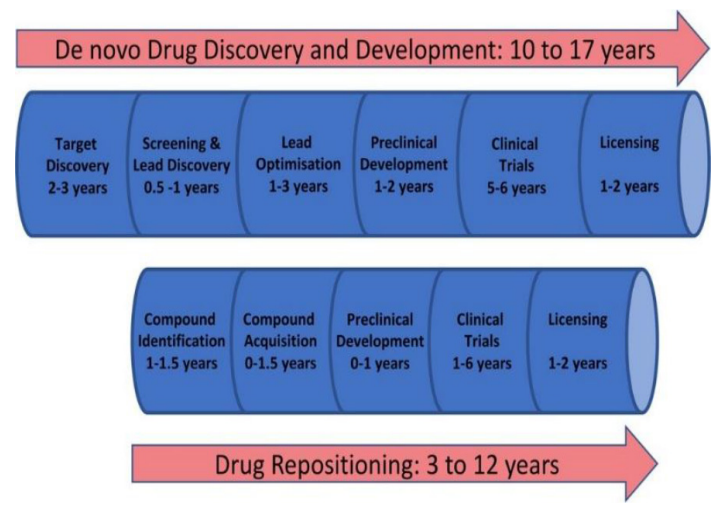

Figure 2: Drug discovery and repositioning pathways. ${ }^{10}$

diseases and many other diseases and disorders (Figure 1$).{ }^{8}$ Drug repurposing is very important to fight the battle against the rapidly spreading infectious diseases, such as HIV, influenza, hepatitis $\mathrm{C}$ virus, Ebola virus, dengue and many other deadly diseases. ${ }^{9}$

Beyond the undeniable economic advantage, the drugs developed by DR approach can quickly enter the clinical trials especially for the diseases which lack specific treatment. Drug repurposing approach serves as a constant source of knowledge in understanding the virus biology and the molecular mechanisms which have been left unexplored. The existing drug molecules with previously unexplored antiviral properties serve as a tool to uncover various viral molecular mechanisms and pathogenesis. Although there are few pitfalls in the drug repurposing approach like difficulty in target identification since the drug may show poly-pharmacology, the effective concentration being higher than the levels which can be achieved in human plasma and hurdles with intellectual property rights, the drug repuposing is still a better approach due to its potential in reducing time and cost of research (Figure 2), its ability to skip preclinical trials and the formulation units would be already setup for large scale production.

There are various drugs which were primarily developed for a disease or disorder, whose antiviral properties are explored to overcome the global threat of emerging and re-emerging viral infectious diseases. Table 1 shows the list of drugs which were repurposed for particular disease along with its original indication for which it was developed.

\section{REPURPOSING IN ZIKA VIRUS INFECTION}

Zika virus (ZIKV) is a mosquito-borne flavivirus, which is associated with severe birth defects and Guillain-Barré syndrome. ZIKA virus infection lack specific antiviral drug or any vaccines for its treatment. The list of FDA approved drugs was screened randomly or a particu-

Table 1: Approved and Candidate Drugs with Repurposing Potential as Antiviral Agents.

\begin{tabular}{|c|c|c|}
\hline Compound & Status/indication & Virus \\
\hline Mycophenolic acid & $\begin{array}{c}\text { Approved/ } \\
\text { immunomodulator }\end{array}$ & ZIKV \\
\hline Daptomycin & Approved/antibacterial & ZIKV \\
\hline Niclosamide & Approved/antiparasitic & ZIKV \\
\hline Azithromycin & Approved/antibacterial & ZIKV \\
\hline Novobiocin & $\begin{array}{l}\text { Approved/ } \\
\text { antibacterial }\end{array}$ & ZIKV \\
\hline Nanchangmycin & Investigational & ZIKV \\
\hline $\begin{array}{l}\text { Hippeastrine } \\
\text { hydrobromide }\end{array}$ & Investigational & ZIKV \\
\hline Sofosbuvir & Approved/antiviral & ZIKV \\
\hline Ribavirin & Approved/antiviral & ZIKV \\
\hline Chloroquine & Approved/antimalarial & ZIKV, MERS- and SARS-CoV \\
\hline Memantine & $\begin{array}{l}\text { Approved/treatment of } \\
\text { Alzheimer's disease }\end{array}$ & ZIKV \\
\hline Prochlorperazine & Approved/antiemetic & DENV \\
\hline Chlorcyclizine & $\begin{array}{c}\text { Approved/ } \\
\text { antihistamine }\end{array}$ & $\mathrm{HCV}$ \\
\hline Manidipine & $\begin{array}{c}\text { Approved/ } \\
\text { antihypertensive }\end{array}$ & JEV, ZIKV, HCMV \\
\hline Favipiravir & Approved/antiviral & EBOV \\
\hline GS-5734 & $\begin{array}{l}\text { Investigational/ } \\
\text { antiviral }\end{array}$ & MERS- and SARS-CoV \\
\hline Imatinib & Approved/anticancer & MERS- and SARS-CoV \\
\hline Chlorpromazine & $\begin{array}{l}\text { Approved/ } \\
\text { antipsychotic }\end{array}$ & MERS- and SARS-CoV \\
\hline $\begin{array}{l}\text { Chlarithromycin/ } \\
\text { Naproxen } \\
\text { + Oseltamivir }\end{array}$ & $\begin{array}{c}\text { Approved/ } \\
\text { antibacterial, } \\
\text { anti-inflammatory } \\
\text { (+antiviral })\end{array}$ & Influenza \\
\hline Nitazoxanide & Approved/antiparasitic & $\begin{array}{c}\text { Influenza, Rotavirus, } \\
\text { Norovirus }\end{array}$ \\
\hline Raltegravir & Approved/antiviral & Herpesvirus \\
\hline $\begin{array}{c}\text { Lopinavir/ } \\
\text { ritonavir } \\
\text { +interferon b-1b }\end{array}$ & Approved/antiviral & MERS-CoV \\
\hline Lopinavir/ritonavir & Approved/antiviral & HPV \\
\hline
\end{tabular}


lar class of compounds was screened based on its activity. ${ }^{2}$ Barrows and colleagues screened a library of 774 FDA-approved drugs for its potential in blocking or inhibiting the infection of human $\mathrm{HuH}-7$ hepatocyte cells by a newly isolated ZIKV strain. Their study identified nearly 24 potential drugs with anti-ZIKV activities, such as ivermectin, mycophenolic acid (MPA) and daptomycin. ${ }^{7}$ The immunosuppressants drug mycophenolic acid and the antibiotic, daptomycin were the promising inhibitors of ZIKA virus replication. ${ }^{11} \mathrm{Xu}$ et al. Screened around 6000 compounds by high-throughput screening approach which included FDA approved drugs, molecules in clinical trials and pharmacologically active compounds. They identified over 100 compounds that inhibited ZIKV-induced caspase 3 activity in SNB-19 cells. ${ }^{12}$ In another study, nanchangmycin, a polyether of bacterial origin, blocked ZIKV infection in different cell lines and in ex vivo midbrain neuron-glia mixed cultures from embryonic mice. ${ }^{13}$ Chloroquine, a commonly used anti-inflammatory, antimalarial agent show antiviral activity against a number of viruses. This candidate also exhibits antiviral activity against ZIKV in Vero, human brain micro vascular endothelial and in neural stem cells. Chloroquine reduces virus production, the number of infected cells and cell death promoted by ZIKV infection without any cytotoxic effect. Sofosbuvir $\left(\mathrm{C}_{22} \mathrm{H}_{29} \mathrm{FN}_{3} \mathrm{O}_{9} \mathrm{P}\right)$ has an antiviral activity against $\mathrm{ZIKV}{ }^{14} \mathrm{Ni}$ closamide and Azithromycin are the most commonly used drugs used in ZIKV treatment in pregnant women and their effective concentration is easily achievable in human plasma. A natural compound Hippeastrine hydrobromide is reported to be a potent inhibitor of ZIKV infection and microcephaly- related effects. Combining drug-target network analysis and functional validation will help in identifying new genes and pathways to develop new antiviral drug molecules for ZIKV infection. Developing new high-throughput, drug-repurposing assays and leveraging existing functional genomics tools against viral replication pathways, is a promising path in the discovery of effective antiviral therapies against ZIKV and other infectious agents.

\section{REPURPOSING IN EBOLA VIRUS INFECTION}

Ebola virus is a filo virus responsible for several outbreaks since its discovery in the late 1970s, but the last one, in 2014-2016, was the most alarming due to its size and spread. Due to the urgent need for an effective Ebola virus treatment, there were additional efforts to study existing drugs as potential anti EVD therapeutic agents which is called as drug repurposing or drug repositioning. The study of favipiravir against Ebola virus has shown good results both in vitro and in vivo. ${ }^{15}$ The ability of Chloroquine to inhibit the Ebola virus has shown promising outcomes in different in vitro studies with various cell lines. ${ }^{16}$ Toremiphene and clomiphene are widely available selective estrogen reuptake modulators approved for the treatment of breast cancer and infertility, respectively. These drugs were found to have antiviral activity by inhibiting the Ebola virus entry in vitro by more than $90 \% .{ }^{17}$ Amiodarone is a well-established and commonly used multi-ion channel blocker, for the treatment of atrial fibrillation and ventricular tachycardia arrhythmias. It has been reported as a potent inhibitor of the Ebola virus in various cell lines. ${ }^{18}$ Among several drugs investigated for anti-Ebola virus activity in vitro and in vivo, Azithromycin was found to be potent in vitro inhibitor of the Ebola virus. ${ }^{19}$ A targeted drug combination approach resulted in the identification of several combinations of drugs which can act synergistically in blocking the Ebola virus entry.

\section{REPURPOSING IN HIV, CMV, HSV AND HCV INFECTION}

HIV/AIDS is one of the worst pandemics in history. According to the World Health Organization, 26 million people have died since 1981 1.6 million in 2012 alone. ${ }^{20}$ In several studies the HIV-1 replication was found to be inhibited by Chloroquine and its hydroxyl analogue hydroxyl Chloroquine. ${ }^{21} \mathrm{HCMV}$ is one of the best examples of host adaptation and of the ability of viruses to subvert, completely, cellular physiological processes in the infected cell. Different DR campaigns identified several approved or investigational drugs with an anti-HCMV mechanism which is different from the existing drugs such as statins, cardiac glycosides, the anti-parasitic drugs emetine and nitazoxanide, kinase inhibitors and the anti-hypertensive drug manidipine. This drug pharmacologically modifies host proteins; the anti-HCMV activity most likely relies on the interference of the drugs with host pathways that are taken away by the virus. ${ }^{22-26}$ The topical treatment with ciclopirox olamine reduces the replication of HSV-1. Zhao et al. reported that the HDAC multi potent inhibitor, suberoylanilide hydroxamic acid which is used for the treatment of cancer reduced the HCV replication in OR6 cells. ${ }^{27}$ Several drugs have shown antiviral activities against $\mathrm{HCV}$ and can be repurposed for the clinical treatment of $\mathrm{HCV}$, such as erlotinib and dasatinib (anticancer drugs), ezetimibe (cholesterol drug) and ferroquine (antimalarial). The two most potent series of $\mathrm{H1}$ - antihistamines, Cyclizine and phenothiazine were found to have anti-HCV activity. ${ }^{28}$

\section{REPURPOSING IN INFLUENZA AND DENGUE}

Influenza virus belongs to the family Orthomyxoviridae and is a pathogen of global public health importance as it causes pandemic disease outbreaks. DR campaigns identified some drugs which are already approved or under clinical evaluation with anti-influenza activity such as BAY 81-8781, dapivirine, naproxen and the antibiotic clarithromycin. A three drug combination of Clarithromycin and naproxen along with oseltamivir showed efficacy in the treatment of severe influenza. ${ }^{29}$ Nalidixic acid and dorzolamide showed efficacy against oseltamivirresistant influenza by an in silico screening specifically targeting mutant viral neuraminidase and, ${ }^{30}$ the most advanced example of DR is the case of the antiparasitic drug nitazoxanide, currently being repurposed for the treatment of influenza. ${ }^{31}$ The kinase inhibitors namely dinaciclib, flavopiridol and PIK-75 were reported to be highly effective against the H7N9 virus with less toxicity. These drugs were also found to be effective against representative strains from the two circulating IAV subtypes, the pdmH1N1 and H3N2. ${ }^{32}$

Dengue is a mosquito-borne viral disease caused by four antigenically distinct serotypes of Dengue Virus (DENV), namely DENV1-4. Dengue is currently the most important arthropod-born viral disease in the world. With the rapid spreading of DENV and the long time required for bringing a new drug on the market, the repurposing of existing seems to be an attractive approach for a rapid therapeutic intervention. ${ }^{33}$ Nelfinavir and other viral protease inhibitors like Lopinavir and Ritonavir were repurposed against Dengue virus infection by computer aided drug design. ${ }^{34}$ As a result of many studies involving Chloroquine in many drug repositioning studies for the dengue virus infection, it was proved to be able to inhibit Dengue Virus Type 2 replication in Vero cells at a dose of $5 \mu \mathrm{g} / \mathrm{ml}$ by plaque assay and qRT-PCR. ${ }^{35}$ Castanospermine is a natural alkaloid is active in vitro against influenza virus, cytomegalovirus, HIV-1 and DENV-1 and in vivo against Herpes Simplex Virus and Rauscher Murine Leukemia Virus. $^{36}$ Dasatinib, Bortezomib and AZD053 (Chemotherapeutic agents), prochlorperazine (Antipsychotic drug), ivermectin, Suramin, Nitazoxanide A(Antiparasitic drugs), dexamethasone, Prednisolone (Steroids), Geneticin, Narasin and Minocycline (Antibiotics) were found to be effective against DENV. ${ }^{33}$

\section{DRUG REPURPOSING FOR OTHER INFECTIOUS DISEASES}

Middle Eastern respiratory syndrome-coronavirus (MERS-CoV) is an emerging virus threat and no specific antiviral agent or vaccine has been 
approved for treating the infected patients. Imatinib mesylate, Toremifene and dasatinib inhibit MERS-CoV and SARS-CoV, while nilotinib inhibits only SARS-CoV. ${ }^{37}$ The dopamine receptor antagonist chlorpromazine and the antimalarial drug Chloroquine were reported to be effective against MERS-and SARS-CoV.

Rift Valley fever virus (RVFV) is a zoonotic arthropod borne emerging infectious disease endemic to sub-Saharan Africa that has recently spread to parts of the Arabian Peninsula. Sorafenib, an orally active multi-kinase inhibitor proved to be most effective against RVFV which was primarily used in the treatment of hepatocellular and renal carcinoma. ${ }^{38}$

\section{CONCLUSION}

With the ever-increasing hurdles in the pharma industry, drug repurposing is the best solution for reducing the risks in the pipeline of new drug discovery. Indeed the economic advantage is remarkable generating up to $\$ 20$ billion in annual sales. Lack of specific therapies with antiviral agent or vaccines is becoming a major medical threat. Repurposing or repositioning the existing FDA approved drugs will serve the need. Understanding the potential of the drug repurposing approach which targets host functions is a time and cost-effective route to develop broad spectrum antivirals. The drug repurposing has already given very positive outcomes with the drugs which are repurposed successfully and also this approach can open new pathways to overcome the challenges with emerging viral threats and antiviral resistance. This strategy already exhibited the feasibility in the development of new anticancer drugs (Such as the antifungal drug itraconazole and its 'second life' as an anticancer drug), but in antiviral drug discovery there are still only a few successful examples (Against influenza, EBOV and MERS-CoV). The drug repurposing approach has given promising candidates in treating various infectious diseases and can be further explored to overcome the drug discovery bottleneck for emerging and re-emerging viral infectious diseases.

\section{ACKNOWLEDGEMENT}

The Authors gratefully acknowledges the scholarship received from Tamil Nadu Pharmaceutical sciences Welfare Trust, Chennai for carrying out research in the area of drug repurposing.

\section{CONFLICT OF INTEREST}

The authors decalre no conflict of interest.

\section{ABBREVIATIONS}

DR: Drug Repurposing; CMV: Cytomegalovirus; HCMV: Human Cytomegalovirus; HCV: Hepatitis C Virus; EVD: Ebola Virus Disease; qRT-PCR: Quantitative Real- Time Polymerase Chain Reaction; RVFV: Rift Valley fever virus; MERS-CoV: Middle East Respiratory Syndrome Coronavirus; SARS-CoV: Severe Acute Respiratory Syndrome Coronavirus.

\section{REFERENCES}

1. Lou Z, Sun Y, Rao Z. Current progress in antiviral strategies. Trends Pharmacol Sci. 2014;35(2):86-102

2. Mercorelli B, Palù G, Loregian A. Drug Repurposing for Viral Infectious Diseases: How Far Are We?. Curr Trends Microbiol. 2018;26(10):865-76.

3. Deenan P, Maria Z. Antiviral drug resistance. BMJ. 1998;317:660

4. Morfin F, Thouvenot D. Herpes simplex virus resistance to antiviral drugs. J Clin Virol. 2003;26(1):29-37.

5. Erice A. Resistance of human cytomegalovirus to antiviral drugs. Clin Microbiol Rev. 1999;12(2):286-97.

6. Ashburn TT, Thor KB. Drug repositioning: identifying and developing new uses for existing drugs. Nat Rev Drug Discov. 2004;3(8):673-83.

7. Cheng F, Murray JL, Rubin DH. Drug Repurposing: New Treatments for Zika
Virus Infection?. Trends Mol Med. 2016;22(11):919-21.

8. Nosengo N. Can you teach old drugs new tricks?. Nature. 2016;534(7607):314-6.

9. Sakurai Y, Kolokoltsov AA, Chen CC, Tidwell MW, Bauta WE, Klugbauer N, et al. Ebola virus: Two-pore channels control Ebola virus host cell entry and are drug targets for disease treatment. Science. 2015;347(6225):995-8.

10. Guha M. Repositioning existing drugs for cancer treatment. Pharm J. 2015;294:7867.

11. Barrows NJ, Campos RK, Powell ST, Prasanth KR, Schott-Lerner G, Soto-Acosta R, et al. A Screen of FDA-Approved Drugs for Inhibitors of Zika Virus Infection. Cell Host Microbe. 2016;20(2):259-70.

12. Xu M, Lee EM, Wen Z, Cheng Y, Huang WK, Qian X, et al. Identification of smallmolecule inhibitors of Zika virus infection and induced neural cell death via a drug repurposing screen. Nat Med. 2016;22(10):1101-7.

13. Yuan S, Chan JF, Den-Haan H, Chik KK, Zhang AJ, Chan CC, et al. Structurebased discovery of clinically approved drugs as Zika virus NS2B-NS3 protease inhibitors that potently inhibit Zika virus infection in vitro and in vivo. Antiviral Res. 2017;145:33-43.

14. Alam A, Imam N, Farooqui A, Ali S, Malik MZ, Ishrat R. Recent trends in ZikV research: A step away from cure. Biomed Pharmacother. 2017;91:1152-9.

15. Sweiti H, Ekwunife O, Jaschinski T, Lhachimi SK. Repurposed Therapeutic Agents Targeting the Ebola Virus: A Systematic Review. Curr Ther Res Clin Exp. 2017;84:10-21.

16. Dowall SD, Bosworth A, Watson R, Bewley K, Taylor I, Rayner E, et al. Chloroquine inhibited Ebola virus replication in vitro but failed to protect against infection and disease in the in vivo guinea pig model. J Gen Virol. 2015;96(12):3484-92.

17. Madrid PB, Chopra S, Manger ID, Gilfillan L, Keepers TR, Shurtleff AC, et al A systematic screen of FDA-approved drugs for inhibitors of biological threat agents. PLoS One. 2013;8(4):e60579.

18. Salata C, Baritussio A, Munegato D, Calistri A, Ha HR, Bigler L, et al. Amiodarone and metabolite MDEA inhibit Ebola virus infection by interfering with the viral entry process. Pathog Dis. 2015;73(5):ftv032.

19. Gielen V, Johnston SL, Edwards MR. Azithromycin induces anti-viral responses in bronchial epithelial cells. Eur Respir J. 2010;36(3):646-54.

20. Michael SK, Eric P. An analysis of FDA-approved drugs for infectious disease: HIV/AIDS drugs. Drug Discov Today. 2014;19(10):1510-3

21. Savarino A, Shytaj IL. Chloroquine and beyond: exploring anti-rheumatic drugs to reduce immune hyperactivation in HIV/AIDS. Retrovirology. 2015;12(1):51.

22. Mercorelli B, Luganini A, Nannetti G, Tabarrini O, Palù G, Gribaudo G, et al. Drug Repurposing Approach Identifies Inhibitors of the Prototypic Viral Transcription Factor IE2 that Block Human Cytomegalovirus Replication. Cell Chem Biol. 2016;23(3):340-51.

23. GardnerTJ, Cohen T, Redmann V, Lau Z, Felsenfeld D, Tortorella D. Development of a high-content screen for the identification of inhibitors directed against the early steps of the cytomegalo virus infectious cycle. Antiviral Res. 2015;113:49-61.

24. Ponroy N, Taveira A, Mueller NJ, Millard AL. Statins demonstrate a broad anticytomegalovirus activity in vitro in ganciclovir-susceptible and resistant strains. J Med Virol. 2015;87(1):141-53.

25. Kapoor A, Cai H, Forman M, He R, Shamay M, Arav-Boger R. Human cytomegalovirus inhibition by cardiac glycosides: evidence for involvement of the HERG gene. Antimicrob Agents Chemother. 2012;56(9):4891-9.

26. Mukhopadhyay R, Roy S, Venkatadri R, Su YP, Ye W, Barnaeva E, et al. Efficacy and Mechanism of Action of Low Dose Emetine against Human Cytomegalovirus. PLoS Pathog. 2016;12(6):e1005717.

27. Zhao F, Liu N, Zhan P, Liu X. Repurposing of HDAC inhibitors toward anti-hepatitis $C$ virus drug discovery: teaching an old dog new tricks. Future Med Chem. 2015;7(11):1367-71.

28. He S, Lin B, Chu V, Hu Z, Hu X, Xiao J, et al. Repurposing of the antihistamine chlorcyclizine and related compounds for treatment of hepatitis $\mathrm{C}$ virus infection. Sci Transl Med. 2015;7(282):282ra49.

29. Hung IFN, To KKW, Chan JFW, Cheng VCC, Liu KSH, Tam A, et al. Efficacy of Clarithromycin-Naproxen-Oseltamivir Combination in the Treatment of Patients Hospitalized for Influenza $A\left(\mathrm{H}_{3} \mathrm{~N}_{2}\right)$ Infection: An Open-label Randomized, Controlled, Phase IIb/III Trial. Chest. 2017;151(5):1069-80.

30. Bao J, Marathe B, Govorkova EA, Zheng JJ. Drug Repurposing Identifies Inhibitors of Oseltamivir-Resistant Influenza Viruses. Angew Chem Int Ed Engl. 2016;55(10):3438-41.

31. Haffizulla J, Hartman A, Hoppers M, Resnick H, Samudrala S, Ginocchio C, et al. Effect of nitazoxanide in adults and adolescents with acute uncomplicated influenza: a double-blind, randomised, placebo-controlled, phase $2 \mathrm{~b} / 3$ trial. Lancet Infect Dis. 2014;14(7):609- 18.

32. Perwitasari O, Yan X, O'Donnell J, Johnson S, Tripp RA. Repurposing Kinase Inhibitors as Antiviral Agents to Control Influenza, a Virus Replication. Assay Drug Dev Technol. 2015;13(10):638-49.

33. Botta L, Rivara M, Zuliani V, Radi M. Drug repurposing approaches to fight Dengue virus infection and related diseases. Front Biosci. 2018;23:997-1019.

34. Bhakat S, Delang L, Kaptein S, Neyts J, Leyssenb P, Jayaprakash V. Reaching beyond HIV/HCV: Nelfinavir as a potential starting point for broad-spectrum 
protease inhibitors against dengue and chikungunya virus. RSC Adv. 2015; 5(104):85938-49.

35. Rolain JM, Colson P, Raoult D. Recycling of chloroquine and its hydroxyl analogue to face bacterial, fungal and viral infections in the $21^{\text {st }}$ century. Int $J$ Antimicrob Agents. 2007;30(4):297-308.

36. Whitby K, Pierson TC, Geiss B, Lane K, Engle M, Zhou Y, et al. Castanospermine, a potent inhibitor of dengue virus infection in vitro and in vivo. J Virol. 2005;79(14):8698-706
37. Dyall J, Coleman CM, Hart BJ, Venkataraman T, Holbrook MR, Kindrachuk J, et al. Repurposing of clinically developed drugs for treatment of Middle East respiratory syndrome coronavirus infection. Antimicrob Agents Chemother. 2014;58(8):4885-93

38. Benedict A, Bansal N, Senina S, Hooper I, Lundberg L, DeLaFuente C, et al. Repurposing FDA-approved drugs as therapeutics to treat Rift Valley fever virus infection. Front Microbiol. 2015;6:676.

Article History: Submission Date : 08-10-2018; Revised Date : 09-11-2018; Acceptance Date : 09-01-2019.

Cite this article: Mani D, Wadhwani A, Praveen TK. Drug Repurposing in Antiviral Research: A Current Scenario. J Young Pharm. $2019 ; 11(2): 117-21$. 